Meta

Journal des traducteurs

Translators' Journal

\title{
Terminologie français-anglais des systèmes experts et des sujets connexes
}

\section{Jacques Lethuillier et Monique C. Cormier}

Volume 35, numéro 4, décembre 1990

URI : https://id.erudit.org/iderudit/003494ar

DOI : https://doi.org/10.7202/003494ar

Aller au sommaire du numéro

Éditeur(s)

Les Presses de l'Université de Montréal

ISSN

0026-0452 (imprimé)

1492-1421 (numérique)

Découvrir la revue

Citer cet article

Lethuillier, J. \& Cormier, M. C. (1990). Terminologie français-anglais des systèmes experts et des sujets connexes. Meta, 35(4), 748-758.

https://doi.org/10.7202/003494ar d'utilisation que vous pouvez consulter en ligne. 


\section{ÉTUDES TERMINOLOGIQUES ET LINGUISTIQUES}

\section{TERMINOLOGIE FRANÇAIS-ANGLAIS DES SYSTĖMES EXPERTS ET DES SUJETS CONNEXES*}

Ce lexique bilingue, non exhaustif, constitue une liste de travail à partir de laquelle est menée une étude méthodique de la terminologie des systèmes experts, qui débouchera sur la production d'un vocabulaire avec définitions. Une deuxième tranche, accompagnée d'un index anglais-français et d'une bibliographie, fera l'objet d'un autre article dans un prochain numéro de la revue.

agenda

agenda

algorithme $\mathrm{A}$

A algorithm

algorithme alpha-bêta

procédure alpha-bêta, procédé alpha-bêta

alpha/beta pruning

algorithme d'appariement

algorithme de filtrage

pattern matcher

match algorithm

algorithme d'inférence

inferencing program

algorithme d'unification

algorithme unificateur

unification algorithm

algorithme de filtrage - Syn. de algorithme d'appariement.

analyse fins-moyens

means-ends analysis

antilogie - Syn. de contradiction.

antécédent — Syn. de prémisse.

arborescence - Syn. de arbre.

arbre

arborescence

tree

arbre de recherche

search tree

$\operatorname{arc}$

arête 
$\operatorname{arc}$

$$
\text { link, edge }
$$

arité

poids

arity

arête - Syn. de arc.

assertion

logical assertion

atome - Syn. de formule.

attachement procédural

valeur active, procédure réflexe

attached procedure

procedural attachment, attached predicate

attribut

attribute

axiomatique - Syn. de système axiomatique.

axiome logique

logical axiom

base de connaissances

knowledge base

$\mathrm{KB}$

base de données - Syn. de base de faits.

base de données déductive

intentional database

IDB

base de faits

registre de mémoire à court terme, base de données, contexte, mémoire de travail factbase

working memory, dynamic knowledge base, short-term memory, global database, database, working memory

base de règles

rule base

but

goal

cadre - Syn. de prototype.

calcul des propositions

logique propositionnelle, logique des propositions propositional logic

PL

calcul des prédicats - Syn. de logique du premier ordre.

calcul symbolique

symbolic computation 
case

$$
\text { rubrique }
$$

slot

chaînage arrière

déduction inverse, raisonnement inductif, raisonnement régressif

backward chaining

inductive reasoning, top-down reasoning, expectations-driven reasoning, goal-driven reasoning, backward search, backward-reasoning

chaînage avant

déduction directe, raisonnement déductif

forward chaining

data-directed inference, data-driven search, forward reasoning, forward search

chaînage mixte

mixed chaining

chemin

path

circonscription

principe de circonscription

circumscription

circuit (dans un graphe)

circuit

clause

clause

clause de Horn

Horn clause

clause résolvante

résolvant, résolvante

resolvent

cogniticien

ingénieur de la connaissance, ingénieur cogniticien

knowledge engineer

condition - Syn. de prémisse.

conditions de déclenchement - Syn. de déclencheur.

conjecture

conjecture

conjectured clause

conjonction

conjunction

connaissance

savoir

knowledge

connaissance de sens commun

common-sense knowledge

commonsense knowledge, common-sense world knowledge, everyday knowledge 
connaissance de surface

surface knowledge

surface level knowledge, shallow surface knowledge

connaissance heuristique

heuristic knowledge

connaissance heuristique - Syn. de connaissance de surface.

connaissances assertionnelles

connaissances déclaratives, connaissances descriptives

factual knowledge

descriptive knowledge, declarative knowledge

connaissances descriptives - Syn. de connaissances assertionnelles.

connaissances déclaratives - Syn, de connaissances assertionnelles.

connaissances opératoires

connaissances procédurales

procedural knowledge

prescriptive knowledge

connaissances procédurales - Syn. de connaissance opératoire.

connecteur logique

opérateur

connector

logical connective

conservation de la cohérence

maintien vérifonctionnel, maintenance de la vérité

truth maintenance system

revision maintenance system, belief revision system, TMS

constante individuelle

individual constant

construction incrémentale

incremental building

conséquence logique

logical consequence

semantic consequence

contexte - Syn. de base de faits.

contradiction

antilogie

contradiction

contrainte

constraint

croyance

belief

cube

cube 
cycle

cycle

cycle d'inférence - Syn. de cycle de base.

cycle d'évaluation-exécution - Syn. de cycle de base.

cycle de base

cycle d'inférence, cycle de moteur d'inférence, cycle d'évaluation-exécution basic inference cycle

cycle de moteur d'inférence — Syn. de cycle de base.

décidabilité

decidability

déclencheur

conditions de déclenchement

trigger

déduction inverse - Syn. de chaînage arrière.

déduction naturelle

natural deduction

défaut

règle par défaut, règle de défaut

default rule

default inference rule, default

délégation

delegation

démon

demon

démonstrateur - Syn. de maquette.

démonstrateur de théorème

programme de démonstration de théorèmes

theorem prover

démonstration automatique de théorèmes

automatic theorem proving

mechanical theorem proving

développement d'un nœud

node expansion

node generation

éditeur de règles

rule editor

encapsulation

data hiding

encapsulation

ensemble de conflit

conflict set

LCS, logical competitor set 
ensemble flou

fuzzy set

épistémologie

gnoséologie, théorie de la connaissance

epistemology

espace d'états

state space

domain

espace de recherche

search space

expert

expert

exploration d'arbre - Syn. de parcours d'un arbre.

exploration en largeur d'abord - Syn. de parcours en largeur d'abord.

explosion combinatoire

combinatorial explosion

expression bien formée - Syn. de proposition composée.

facette

facet

fait initial

initial input

FBF - Syn. de proposition composée.

feuille

noud terminal

terminal node

tip node, leaf

filtrage flou

fuzzy matching

fonction

forme fonctionnelle

functional form

fonction d'évaluation

interestingness function

heuristic search function, heuristic evaluation function, heuristic estimation function, heuristic function

formalisme de représentation de la connaissance

knowledge representation formalism

forme clausale

cllausal form

forme conjonctive normale 
conjunctive normal form

$\mathrm{CNF}$

forme disjonctive normale

disjunctive normal form

forme fonctionnelle - Syn. de fonction.

forme normale prénexe

prenex normal form

forme propositionnelle - Syn. de proposition composée.

forme prédicative - Syn. de prédicat.

forme-objet - Syn. de prototype.

formule

atome, formule atomique

atomic formula

atomic sentence, single proposition

formule atomique - Syn. de formule.

formule bien formée - Syn. de proposition composée.

formule logique composée - Syn. de proposition composée.

fragment de connaissance

parcelle de connaissance, granule de connaissance

chunk of knowledge

frame - Syn. de prototype.

généralisation

generalization

générateur de plans

générateur de plans d'actions, planificateur

planner

générateur de plans d'actions - Syn. de générateur de plans.

générateur de systèmes experts

GSE, logiciel de développement de systèmes experts, outil de développement expert system shell

knowledge engineering tool, expert system building tool, expert system generator, knowledge-based system shell

génération de plans

planning

génie cognitif

knowledge engineering

gnoséologie - Syn. de épistémologie.

grammaire indépendante du contexte

context-free grammar

granularité (de la connaissance) - Syn. de modularité (des règles, de la connaissance).

granule de connaissance — Syn. de fragment de connaissance. 


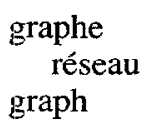

graphe complet complete graph

graphe connexe connected graph

graphe ET/OU

AND / OR graph

graphe non orienté undirected graph

graphe orienté

directed graph digraph

GSE - Syn. de générateur de systèmes experts.

héritage

inheritance

héritage multiple

multiple inheritance

heuristique

heuristic

hypothèse

hypothesis

hypothèse du monde fermé

closed world assumption

\section{CWA}

implication

implication

if ... then, strict implication, modal implication

implication minimale

stratégie d'implication minimale, stratégie de moindre engagement

least commitment

induction

induction amplifiante, raisonnement inductif

inductive inferencing

induction, inductive inference

induction amplifiante - Syn. de induction.

inférence - Syn. de raisonnement.

inférence - Syn. de inférence logique.

inférence logique

inférence, pas de raisonnement

logical inference 
ingénieur cogniticien - Syn. de cogniticien.

ingénieur de la connaissance - Syn. de cogniticien.

instanciation

instantiation

interprétation

interpretation

interprète - Syn. de moteur d'inférence.

interpréteur - Syn. de moteur d'inférence.

interpréteur des faits et des règles - Syn. de moteur d'inférence.

langage acteur

actor language

langage applicatif

langage fonctionnel

applicative language

functional language

langage fonctionnel - Syn. de langage applicatif.

langage objet

object language

langage orienté objet

object-oriented language

langage propositionnel

propositional language

LIPS

LIPS

Logic Inference Per Second

littéral

literal

logiciel de développement de systèmes experts - Syn. de générateur de systèmes experts.

logique

logic

théorie logique

logique à plusieurs valeurs - Syn. de logique multi-valuée.

logique aléthique - Syn. de logique du possible.

logique catégorique

exact logic

two-valued logic

logique des croyances - Syn. de logique épistémique.

logique des propositions - Syn. de calcul des propositions.

logique des prédicats - Syn. de logique du premier ordre. 
logique du possible

logique aléthique

alethic modal propositional logic

logique du premier ordre

logique prédicative, calcul des prédicats, logique des prédicats

predicate logic

FOPC, FOPL, FOL, first-order logic, predicate calculus

logique déontique

deontic logic

logique épistémique

logique des croyances

epistemic logic

logique floue

fuzzy logic

fuzzy set theory

logique formelle

formal logic

logique mathématique

logique moderne, logistique, logique symbolique

mathematical logic

symbolic logic

logique modale

modal logic

logique multi-valuée

logique à plusieurs valeurs

multivalued logic

inexact logic, many-valued logic

logique non monotone

logique révisable

non monotonic logic

logique prédicative - Syn. de logique du premier ordre.

logique propositionnelle - Syn. de calcul des propositions.

logique révisable - Syn. de logique non monotone.

logique temporelle

temporal logic

machine déductive - Syn. de moteur d'inférence.

maintenance de la vérité - Syn. de conservation de la cohérence.

maintien vérifonctionnel - Syn. de conservation de la cohérence.

maquettage

prototypage

prototyping

maquette

démonstrateur, prototype, pré-système 
prototype

mécanisme d'inférence - Syn. de moteur d'inférence.

mécanisme d'interprétation - Syn. de moteur d'inférence.

mécanisme de contrôle - Syn. de moteur d'inférence.

mécanisme de déduction - Syn. de moteur d'inférence.

mémoire de travail - Syn. de base de faits.

métalangage

metalanguage

métarègle - Syn. de règle de stratégie.

méthode de résolution - Syn. de résolution.

modularité (des règles, de la connaissance)

granularité (de la connaissance)

granularity

module d'édition de la connaissance

knowledge editor

modus ponens

règle de détachement

modus ponens

modus tollens

raisonnement par l'absurde

modus tollens

monotone

monotonic

monotonie

monotonicity

moteur d'inférence

machine déductive, interpréteur, mécanisme de déduction, mécanisme d'inférence, mécanisme d'interprétation, interpréteur des faits et des règles, mécanisme de contrôle, interprète

inference engine

control program, rule interpreter

négation par échec

negation as failure

nœud

sommet

node

vertex

nœud terminal — Syn. de feuille.

notation préfixée

prefix notation

objet

object 
OOP - Syn. de programmation orientée objet.

opérateur - Syn. de connecteur logique.

opérateur temporel

tense modifier

tense operator

outil de développement - Syn. de générateur de systèmes experts.

Jacques Lethuillier et Monique C. Cormier

Université de Montréal

* Projet de recherche réalisé grâce à une aide financière de IBM Canada. 